\title{
Measuring the Dynamic Mechanical Characteristics of Japanese Food Materials against Small Impact Force
}

\author{
Ryosuke Araki $^{\text {a, }}$, Kyohei Irisa ${ }^{\mathrm{a}}$, Kazuhide Watanabe $^{\mathrm{a}}$, Naoki Miyashita ${ }^{\mathrm{a}}$, Akihiro Takita ${ }^{\mathrm{a}}$, Yusaku Fujii ${ }^{\mathrm{a}}$ \\ ${ }^{\mathrm{a}}$ School of Science and Engineering, Gunma University \\ *Corresponding Author: t10306004@gunma-u.ac.jp
}

\begin{abstract}
The dynamic mechanical characteristics against small impact force of konjak, one of popular Japanese foods, were measured in this study. There are many studies discussing the sensory characteristics of food materials, however few of them measure the characteristics numerically and accurately; therefore we have done the experience and show the characteristics. Konjak is flexible and slimy food material. We thought it is like rubbers and measured the response against small impact force. To measure the precise dynamic mechanical force, the levitation mass method (LMM) is used. In LMM, a pneumatic linear bearing is used to reduce friction to almost zero, and an optical interferometer is used. The acceleration, velocity, position, and inertial force of the mass are calculated from the measured time-varying frequency due to Doppler effect. LMM is used to only measure the dynamic forces of the mass before, but we utilize it to test the mechanical characteristics of food materials. Konjak has the characteristics of viscoelasticity. We reveal the numerical and logical characteristics of konjak.
\end{abstract}

Keywords: Levitation mass method, konjak, material testing.

\section{Introduction}

The evaluation of the mechanical properties of materials is important. There are many mechanical properties, and force is the most important among them. However, it is difficult to measure a dynamic force that changes with time, and a suitable method for evaluating it is yet to be established (1). In previous works, Dr. Y. Fujii proposed the levitation mass method (LMM) (2-7), in which a mass is levitated using a pneumatic linear bearing, which makes the friction acting on the mass negligible. The inertial force of the mass can therefore be measured precisely. In this method, an optical interferometer is used, as shown Figure 1, and only the time-varying beat frequency $f_{\text {beat }}$ is measured; all the other quantities such as the velocity $v$, position $x$, acceleration $a$, and force $F$, are subsequently calculated.

Although the mechanical properties of traditional food materials have been discussed in many studies (8), few of them discussed the numerical and logical characteristics. Most of them focused on the sensory properties of food materials, such as appearance, smell, taste, and texture. Konjak is a traditional Japanese food that is very popular in Japan. It is flexible and slimy, and resembles rubber. In this paper, we propose numerical and logical characteristics of konjaks against small impact force.

\section{Materials and Method}

Figure 2 is a schematic of the experimental setup for evaluating the dynamic properties of the material. A pneumatic linear bearing (GLS08A50/25-2571; NSK Co., Ltd., Japan) was used to reduce the friction of the mass. The tested material was attached to the base by means of its viscosity. An initial force was manually applied to the

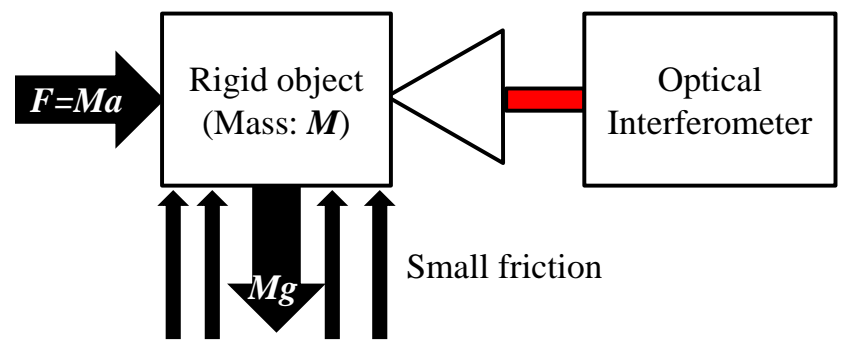

Fig.1. Principle of LMM 
moving part of the pneumatic bearing, which had a mass of $31.11 \mathrm{~g}$. A corner cube prism (CC) and a dumper with a rounded tip (radius of curvature: $10 \mathrm{~mm}$ ) were attached to the moving part. In this study, konjaks were the test materials. All the cut konjaks had square cross sections of side $20 \mathrm{~mm}$. Three different thicknesses, namely 3, 5, $8 \mathrm{~mm}$, were used. The uncertainty of each thickness was $1 \mathrm{~mm}$. In this test, seven collisions were manually executed for each material using various initial velocities of the moving part.

A Zeeman-type two-frequency He-Ne laser was used as the light source. $S_{\text {rest }}$ and $S_{\text {beat }}$ are the intensities of the rest and beat beams, respectively. $f_{\text {rest }}$ is the frequency of $S_{\text {rest }}$, and $f_{\text {beat }}$ is the difference between the frequencies of $S_{\text {beat }}$ and it was measured at the interference fringe. The fringe was observed using the photo diode (PD). $f_{\text {beat }}$ was changing around $f_{\text {rest }}$, which had an average value of approximately $1.7 \mathrm{MHz}$. The change was dependent on the velocity of the moving part. A digitizer (NI PCI-5105; National Instruments Corp., USA) was used to continuously measure $S_{\text {beat }}$ and $S_{\text {rest }}$ $60,000,000$ times per second without dead time.

The measurements initiated by a sharp trigger generated by a light switch composed of a digital-to-analog converter (DAC), laser diode (LD), and photo diode (PD). Both the initial time $t=0$ and the initial position $x=0$ coincided with the first detection of the reaction force of the material.

The force applied to the moving part $F_{\text {mass }}$ is, by definition, the product of the mass of the moving part $M$ and the acceleration $a$. The acceleration of the moving part $a$ was calculated by differentiating the time-varying velocity. The velocity of the moving part $v$ can be calculated from the Doppler frequency using the following equation:

$$
\begin{gathered}
v=\lambda_{\text {air }} f_{\text {Doppler }} / 2, \\
f_{\text {Doppler }}=-\left(f_{\text {beat }}-f_{\text {rest }}\right),
\end{gathered}
$$

where $\lambda_{\text {air }}$ is the length of the laser beam wave in the air, $f_{\text {beat }}$ is the beat frequency, and $f_{\text {rest }}$ is the rest frequency. $\lambda_{\text {air }}$ was approximately $630 \mathrm{~nm}$ in the test. $f_{\text {beat }}$ is the difference between the frequency of the signal beam and the reference beam. $f_{\text {rest }}$ is the beat frequency when the moving part is at rest. The inertial force can therefore be calculated from the beat frequency $f_{\text {beat }}$ with high accuracy.

\section{Result}

Figure 3 shows the data processing procedure. In this experiment, an optical interferometer was used, and only the beat frequency $f_{\text {beat }}$ and rest frequency $f_{\text {rest }}$ were therefore accurately obtained. First, the two frequencies were converted to the velocity $v$ using equation (1). The position $x$ was then obtained by integrating $v$, and the acceleration $a$ was obtained by differentiating $v$. Further, the force $F$ was calculated by the equation $F=m a$. The initial position $x=0$ and time $t=0$ were determined when the force $F$ was $0.5 \%$ of the maximum force $F_{\text {max }}$.

Figure 4 is measured impact force $F$ as a function of

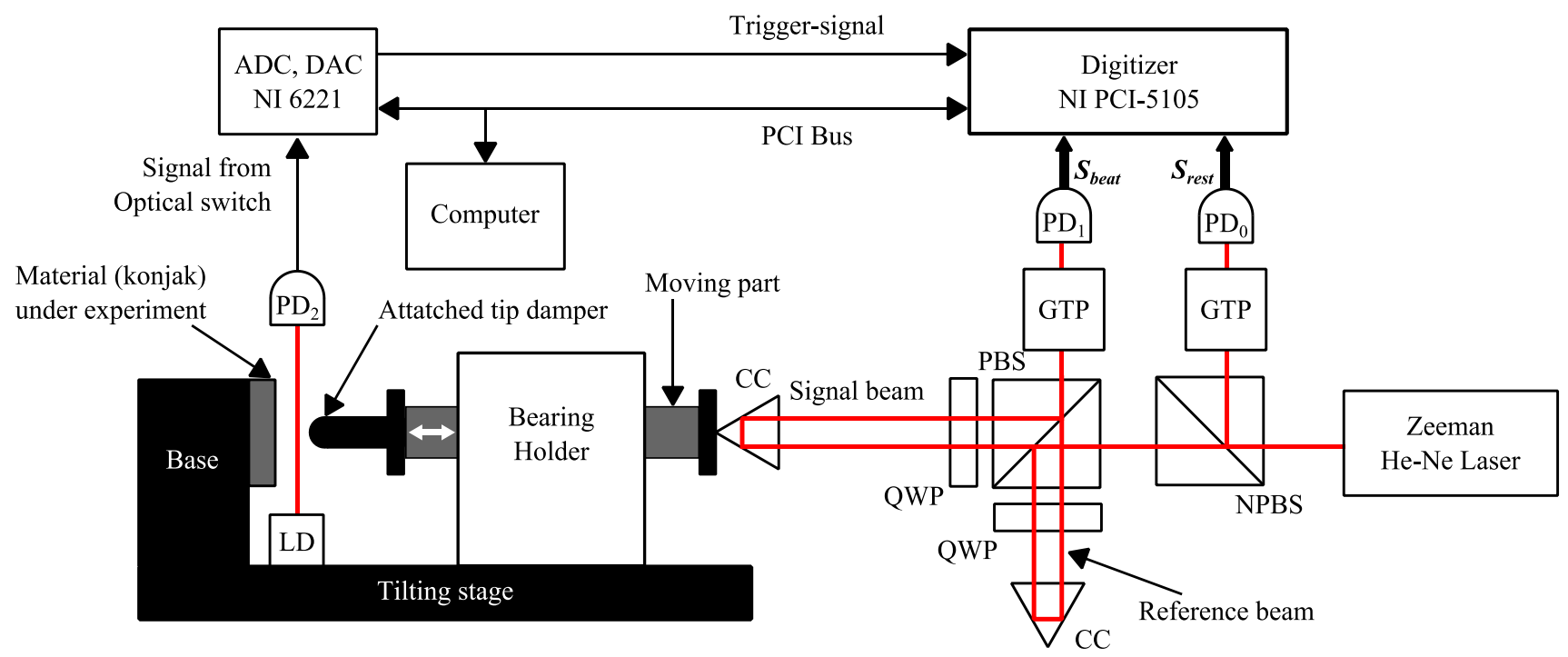

Fig.2. Experimental Setup. PBS $=$ Polarizing beam splitter, NPBS $=$ Non-polarizing beam splitter, QWP $=$ Quarter wave plate, $\mathrm{CC}=$ Cube corner prism, $\mathrm{GTP}=$ Gland-Thompson prism, $\mathrm{PD}=$ Photo diode, $\mathrm{ADC}=$ Analog-to-digital Converter, DAC $=$ Digital-to-analog converter, and LD = Laser diode. 
the position $x$. In the figure, the thickness of the materials is $3 \mathrm{~mm}$, and the maximum value of each selected data is approximately $0.8 \mathrm{~N}$. The hysteresis caused by the viscosity of the material is clearly shown.

Figures 5 and 6 are the graphs of the measured force against the position of konjak $\mathrm{A}$ and $\mathrm{B}$, respectively. The hysteresis is clearly observed in the graphs.

\section{Discussions}

Figure 4 shows that the parameters do not change for different types of konjaks of the same thickness. From Figures 5 and 6 , it can be observed that the thicker the material, the farther the position. The tendencies of the plotted points are almost the same in the three figures, although the points for $8 \mathrm{~mm}$ are not similar to those for 3 and $5 \mathrm{~mm}$ in Figure 5. This was due to the uncertainty of the thickness.

Figure 7 shows the maximum force $F_{\max }$ as a function of half width $W_{h v}$ for all the konjaks. The points of $W_{h v}$ are inversely proportional to the maximum force
$F_{\max }$

Figures 4 and 5 show the force $F$ as a function of $x$. The hysteresis can be clearly observed in these figures. By using $E=1 / 2 m v^{2}$, the energies of the moving part before collision $\left(E_{0}=1 / 2 m v_{0}^{2}\right)$ and after collision $\left(E_{1}=1 / 2 m v_{1}^{2}\right)$ were calculated. The graph of $E_{0}$ against the absorbed ratio $\left(1-E_{1} / E_{0}\right) \times 100$ is shown in Figure 8, which reveals that the parameters in the figure are independent of the type of konjaks. The rate of decrease $\left(1-E_{1} / E_{0}\right) \times 100$ is independent of the type of konjak, but dependent on the thickness.

These characteristics indicate that konjaks are viscoelastic objects. They can also absorb approximately $50 \%$ of small impact shocks.

\section{Conclusions}

Over all, the dynamic mechanical reaction of food materials such as konjaks can be accurately and numerically measured using LMM.
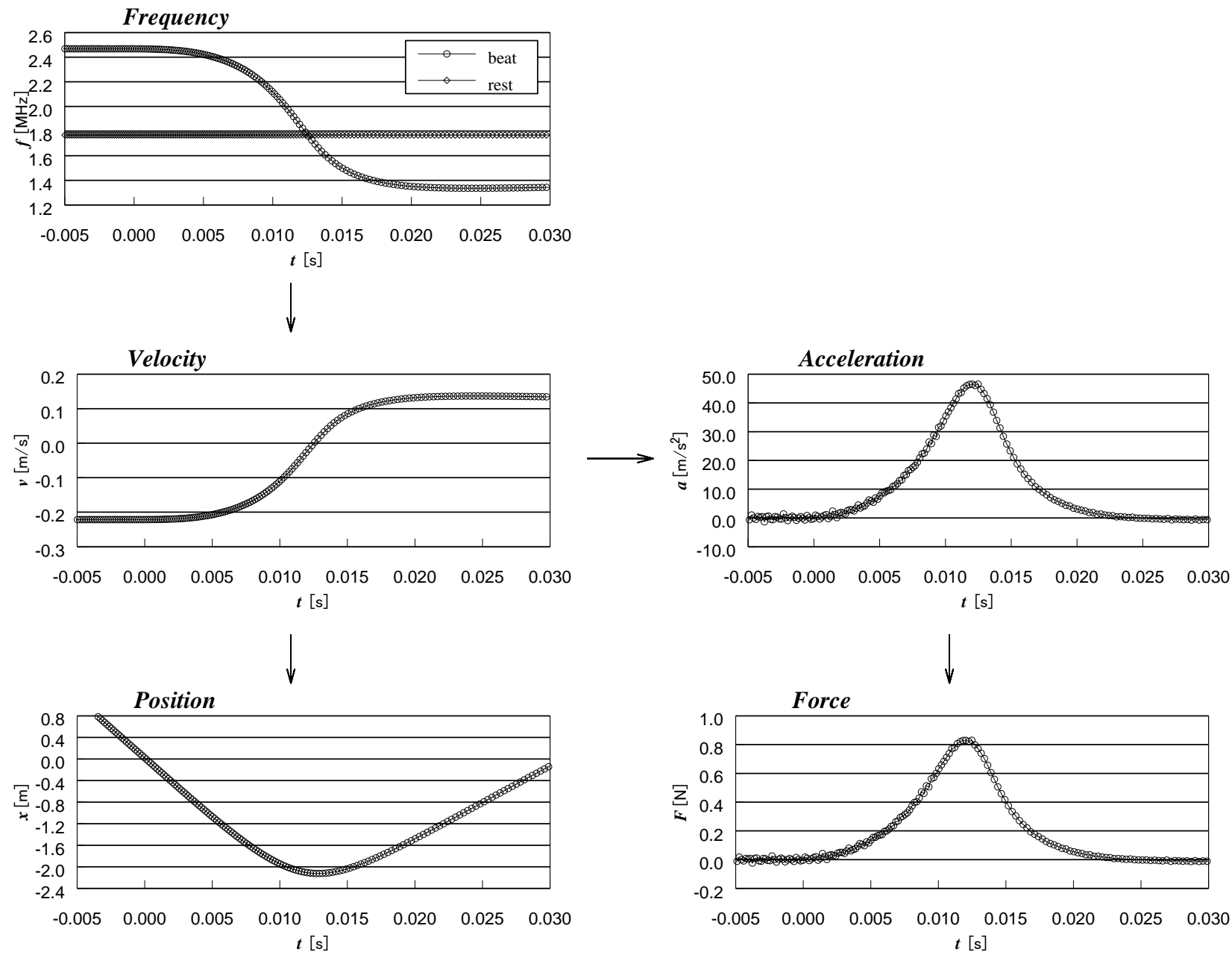

Fig. 3. Data processing procedure of this experiment. 


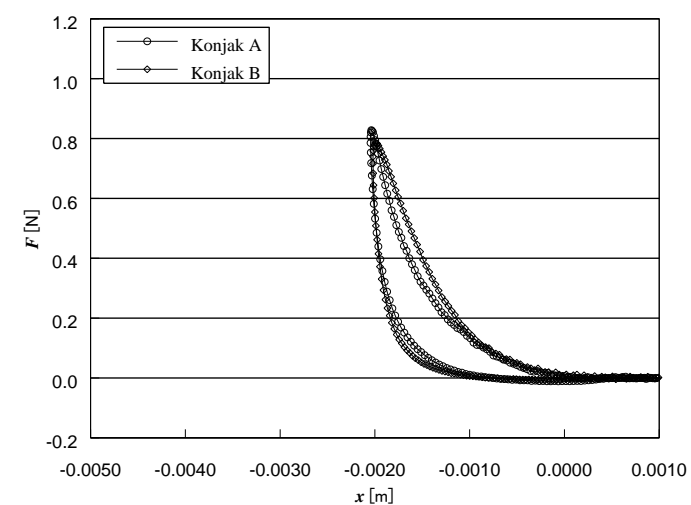

Fig. 4. Measured impact force $F$ against position $x$ for all materials.

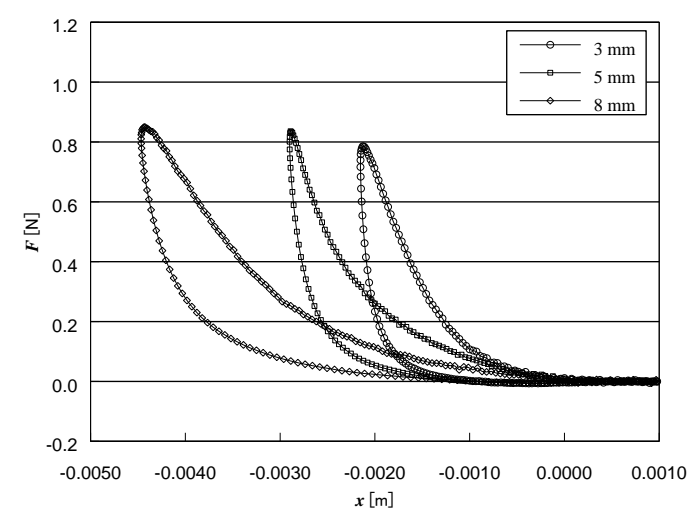

Fig. 6. Measured impact force $F$ against position $x$ for konjak B (thickness: $3 \mathrm{~mm}, 5 \mathrm{~mm}, 8 \mathrm{~mm}$ )

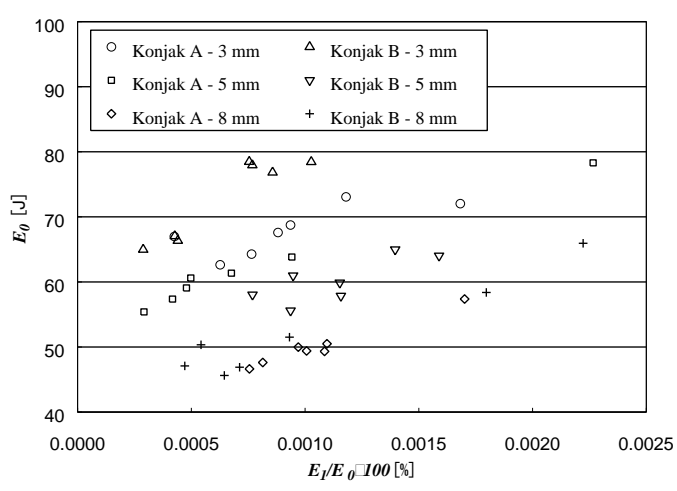

Fig. 8. Decreasing rate due to collision $E_{1} / E_{0} \times 100$ against the kinetic energy of the moving part before collision $E_{0}=1 / 2 m v_{0}^{2}$, where $E_{1}$ is defined by following equation: $E_{1}=1 / 2 m v_{1}^{2}, v_{0}$ is the average of the initial velocities of 50 points, and $v_{1}$ is the average of their terminal velocities.

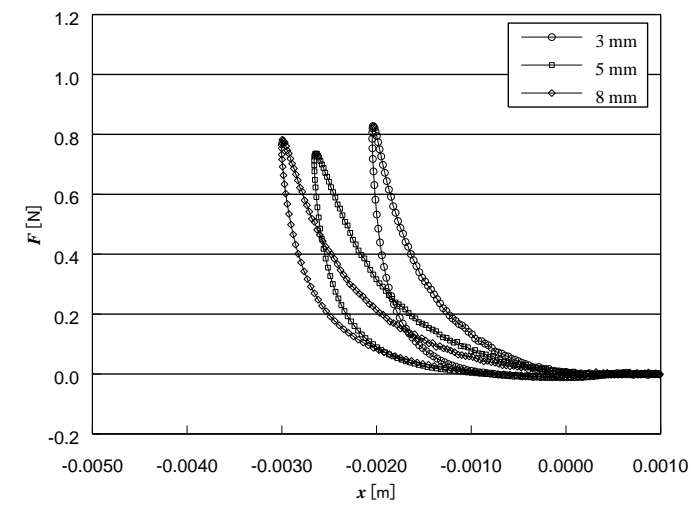

Fig. 5. Measured impact force $F$ against position $x$ for konjak A (thickness: $3 \mathrm{~mm}, 5 \mathrm{~mm}, 8 \mathrm{~mm}$ )

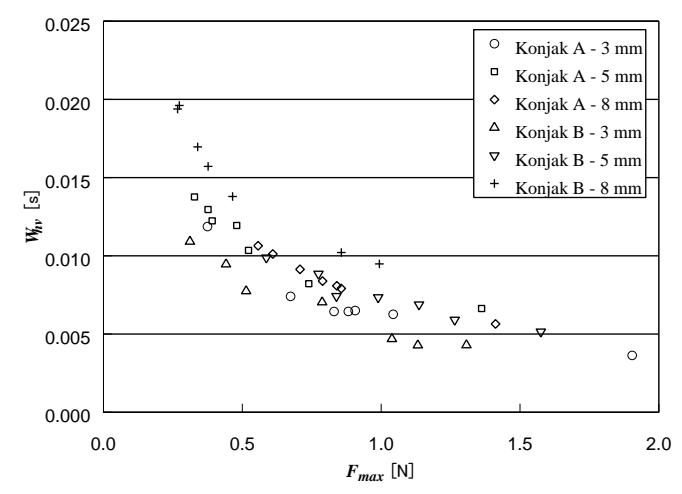

Fig. 7. Half width $W_{h v}$ against maximum force $F_{\text {max }}$.

\section{Acknowledgment}

This work was supported in part by a research-aid fund of the NSK Foundation for the Advancement of Mechatronics (NSK-FAM) and the Grant-in-Aid for Scientific Research (B) 24360156 (KAKENHI 24360156)

\section{References}

(1) J.P.Hessling : "Models of dynamic measurement error variations of material testing machines," Mechanical Systems and Signal Processing, vol. 23, no. 8, pp.2510-2518, 2009.

(2) Y. Fujii : "The Levitation Mass Method: A Precision Mass and Force Measurement Technique," International Journal of Precision Engineering and Manufacturing, vol. 9, no. 3, pp. 46-50, 2008.

(3) Y. Fujii : "Impact force measurement using an inertial mass and a digitizer," Measurement Science and Technology, vol. 17, no. 4, pp. 863-868, 2006. 
(4) Y. Fujii : "Method for evaluating material viscoelasticity," Review of Scientific Instruments, vol. 75, no. 1, pp.119-123, 2004.

(5) Y.Fujii : "Method for generating and measuring the micro-Newton level forces," Mechanical Systems and Signal Processing, vol. 20, no. 6, pp. 1362-1371, 2006.

(6) Y.Fujii : "Measurement of force acting on a moving part of a pneumatic linear bearing," Review of Scientific Instruments, vol. 74, no. 6, 3137-3141, 2003.

(7) Y.Fujii : "Microforce materials tester," Review of Scientific Instuments, vol. 76, no. 6, pp. 065111 1-7, 2005.

(8) Ohisa : "Comparison of Hardness of Cooked Inaniwa-udon and Hardness of Other Wheat Noodles(Japanese)," Nippon Shokuhin Kagaku Kogaku Kaishi, vol. 52, no. 11, pp. 522-527 